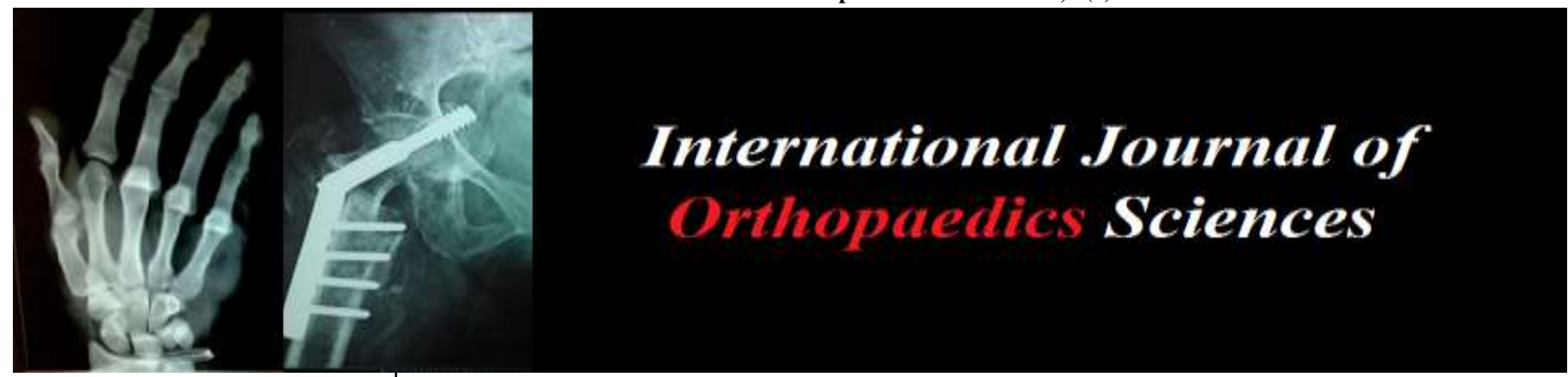

E-ISSN: 2395-1958

P-ISSN: 2706-6630

IJOS 2020; 6(4): 885-890

(C) 2020 IJOS

www.orthopaper.com

Received: 17-08-2020

Accepted: 27-09-2020

\section{Ashish Jain}

Assistant Professor, Department of Orthopaedics, Hind Institute of Medical Sciences, Barabanki, Uttar Pradesh, India

Deepak Srivastava

Associate Professor, Department of Orthopaedics, Hind Institute of Medical Sciences, Barabanki, Uttar Pradesh, India

AN Mishra

Professor, Department of Orthopaedics, Hind Institute of Medical Sciences, Barabanki, Uttar Pradesh, India
Corresponding Author:

Ashish Jain

Assistant Professor, Department of Orthopaedics, Hind Institute of Medical Sciences, Barabanki, Uttar Pradesh, India

\section{Effects of targeted back muscle exercises in reducing thoracic kyphosis in patients of hyperkyphosis}

\author{
Ashish Jain, Deepak Srivastava and AN Mishra
}

DOI: https://doi.org/10.22271/ortho.2020.v6.i4m.2434

\section{Abstract}

Increased thoracic kyphosis (hyperkyphosis) is well known entity especially in elderly population may be responsible for multiple morbidity and needs a non-pharmacological preventive strategy due to which muscle strength training has been routinely advised. Eighty patients were included in study and divided into two groups. One group received institutional and home-based exercise regimen and another group was administered the routine pharmacological regimen. Besides demographic variables, clinical (occiput to wall distance, pain, ODI score and spinal flexibility) and radiological parameters (Cobb's angles of thoracic kyphosis, lumbar lordosis, and thoracic kyphosis in best erect posture) were assessed before and after the end of 3-month duration. The data between both the groups was comparable but the study group showed marked improvement at the end of study (mean difference of $1.8^{\circ}$ ) and significant improvement in clinical parameters of pain, ODI score, occiput to wall distance and spinal flexibility. The other values were insignificantly affected. We find that exercise regimen positively impacts and significantly improves the thoracic kyphosis, clinical and functional status of patients who have hyperkyphosis but undergo a targeted muscle training programme for back muscles.

Keywords: Hyperkyphosis, thoracic kyphosis, Back extension strength

\section{Introduction}

Hyperkyphosis is usually defined as an excessive anterior concavity of the thoracic spine usually more than 45 degrees ${ }^{[1]}$. It is a common occurrence in patients presenting to spine outpatient department in our area and is more prevalent in cases of spinal tuberculosis and Senile osteoporosis. It is estimated, however, to affect $20 \%$ to $40 \%$ of older adults, worldwide ${ }^{[2,3]}$. Respiration is significantly affected in this condition due to reduced pulmonary function ${ }^{[4]}$ and is usually associated with poorer outcomes in activities of daily living performance ${ }^{[3,5]}$, quality of life ${ }^{[3]}$, and a higher prediction of mortality which might be independent of underlying spinal osteoporosis ${ }^{[2]}$.

Hyperkyphosis can be present due to numerous causes which are varied in nature. Vertebral body wedging, a persistent poor posture, impaired motion of spinal extension, diminished strength of back extensor muscle strength ${ }^{[2,6,7]}$ is other commonly cited potential causes of age-related hyperkyphosis. Increased kyphosis in thoracic spine attributed to anterior displacement of centre of gravity and is associated with muscle impairments and altered gait [8]. This may lead to a poor function of lower limbs, which is also important to understand as subsequent mobility impairment and loss of independence may be predicted or prevented in such individuals ${ }^{[9]}$. All these concerns warrant a higher attention on hyperkyphosis, which till date is only recently becoming recognized by health care providers as a health concern with no significant standard of care ${ }^{[10]}$. A pharmacological treatment based on osteoporosis may have a limited role in preventing hyperkyphosis, as only a third of patients with hyperkyphosis have evidence of underlying vertebral fractures ${ }^{[11]}$. Studies done in other part of the world have subjected patients of hyperkyphosis to other treatment protocols such as targeted spinal muscle strength and back extensor muscle training with good results and modest improvement or arrest in kyphosis. This was combined with orthosis and other technological tools with some success. Moreover, a systematic review including seven randomized controlled trials reported that exercise interventions targeting back extensor muscle strength resulted in modest improvements in clinical measures of kyphosis ${ }^{[1,12,13,14]}$. 
As most of these studies have studied varied parameters with very few of them including radiological measurement of Thoracic kyphosis and Lumbar lordosis in post-intervention group, this study was taken to study the clinic-radiological effect of targeted spinal extension strengthening protocol in patients of our geographical area as a management of Hyperkyphosis.

\section{Methods}

A total of 80 patients who reported to Department of Orthopaedics at Hind Institute of Medical Sciences, Barabanki were included in the study which was carried out for 3 months for each of the patients. This study was done between 2018 January to August 2020.

The inclusion criteria consisted of patients who were clinically hyperkyphotic and had a thoracic kyphosis more than 40 degrees of Cobb's angle in erect Xray on lateral view. Patients who had a concurrent neoplasm, inflammation, or infection and who had indications for urgent surgery (cauda equina syndrome or progressive motor deficit), previous history of spinal surgery, current pregnancy or early postpartum period (6 months), and coexisting medical conditions (severe central or foraminal spinal stenosis gross structural abnormalities, such as spondylolisthesis or scoliosis, ankylosing spondylitis, spinal fracture, limb fracture, visual loss and spinal tumour) were excluded from the study. Written informed consent was obtained from all patients prior to data collection. The study was passed through the ethical committee of our institution.

Patients who were able to attend Physiotherapy for 10 sessions ( 5 times/week for 2 weeks) followed by a strict and compliant home protocol for 10 weeks and who were willing to adhere to the protocol were included in the study.

Forty patients were assigned to the study group and were scheduled for the exercise programme. The control group was composed of forty age- and sex-matched patients and received no targeted exercise regimen but other conservative treatment was administered. In the study group, initial sessions were institution based for 2 weeks and consisted of 10 training sessions. Each of these sessions was performed in presence of one responsible attendant of the patient. The spinal extension and muscle training exercises were demonstrated by a physiotherapist in our supervision in the first few sessions and then followed up in the subsequent sessions. These regimens were tailored to each patient based on the existing muscle strength and capacity of individuals in terms of repetitions and sets, they were advised to. These exercises consisted of spinal extension exercises in supine and prone position, Mc Kenzie exercises, Scapular and wall pushing exercises, arm raises, Posture training, modified Hatha yoga and prone extension with or without weight.

Home-based exercise programs consisted also of isometric and isotonic strengthening exercises for the paraspinal and abdominal muscles and stretching exercises for the back extensors, hamstrings, and calf muscles. This exercise programme was prescribed for 10 weeks. Attendants of patients were followed up telephonically and randomly asked for video demonstration of their home-based exercises to rule out compliance issue. There were no restrictions against medical treatments patients were taking during the outpatient evaluation. Other co-interventions were not allowed during the treatment period.

Study variables pertained to demography, clinical and radiological parameters

The demographic variables consisted of age, gender, working level (Sedentary, Moderate or Heavy worker), BMI and duration of symptoms. The clinical parameters consisted of pain (measured by VAS), ODI score, Occiput to wall distance and Modified Schoeber test for spinal mobility The radiological parameters consisted of Thoracic kyphosis on erect Xray, Lumbar lordosis on erect Xray and Thoracic kyphosis on erect extension.

The clinical and radiological parameters were assessed at the starting of study and at the end of 3 months in each of the groups.

\section{Results}

Eighty patients (study group, $\mathrm{n}=40$ and control group, $\mathrm{n}=40$ ) with a mean age of $67.19+/-22.14$ years were included in the study. None of the patients had any issues regarding issues of compliance or adherence. There was no exit or expiry in any of the groups. In the study group $41.25 \%$ of patients $(n=33)$ were male and rest of the patients $(n=47)$ were female. In the control group, $43.75 \%$ of patients $(n=35)$ were male and rest of the patients were female $(n=45)$. The mean symptom duration was $8.6 \pm 9.7$ years. Age wise there was no significant difference $(p=0.9167)$ between the two groups. Study group had a mean of $68.16+/-19.45$ years while the control group had a mean of $66.22+/-24.28$ years.

BMI between both the groups was comparable. Mean BMI score of study group was $21.8(\mathrm{p}=0.19)$ and control group was $22.14(\mathrm{p}=0.24)$. Most of the patients in both the groups were of sedentary activity level owing to increased age and showed no significant difference between both the groups. Table 1 shows the patients' complete demographic and clinical features. There were no statistically significant differences between the groups in terms of age, sex, body mass index, symptom duration, or working status $(\mathrm{p}>0.05)$ (Table 1$)$.

The clinical data depicted a significant improvement in the study group as compared to the control group. Comparison at the starting of the study showed the pain intensity (measured on VAS), ODI score, Lumbar flexibility (as measured by modified Schoeber test) and Clinical hyperkyphosis as measured by Occiput to wall distance to be comparable between both (study and control) the groups.

The radiological data showed comparative data in both the groups at the beginning of the study. Thoracic kyphosis in the study group was $49.12^{\circ}+/-4.53^{\circ}$ which was comparable to the values of thoracic kyphosis in the control group $\left(48.75^{\circ}\right.$ $\left.+/-3.75^{\circ}\right)$. The value of thoracic kyphosis improved to $47.3^{\circ}$ $+/-5.12^{\circ}$ after the exercise regime at the end of the 3 months (chart 1). The mean reduction in angle was 1.8 degrees which was statistically significant $(\mathrm{p}=0.019)$. The control group had minimal reduction in angles $(0.7$ degrees, $\mathrm{p}=0.16)$. The mean value of thoracic kyphosis at the end of study was $48.05^{\circ}+/-$ $4.23^{\circ}$. The Lumbar lordosis also was comparable (study = $34.05^{\circ}=/-4.3^{\circ}$, control: $32.75^{\circ}+/-3.7^{\circ}$ ), the values in study group improved to $32.2^{\circ}+/-3.7^{\circ}$, but this result was just short of being statistically significant $(\mathrm{p}=0.056)$. The control group also improved to $31.15^{\circ}+/-3.4^{\circ}$ and this was also statistically insignificant $(\mathrm{p}=0.016)$. The values of thoracic kyphosis at best erect posture were showed no significant change either between the study and control groups ( study $=45.8^{\circ}+/-4.85^{\circ}$, control $=44.75^{\circ}+/-6.3^{\circ}$ ) or with values at the end of study (study: $44.5^{\circ}+/-4.8^{\circ}$, control: $44.05^{\circ}+/-6.1^{\circ}$ ). 


\subsection{Charts}

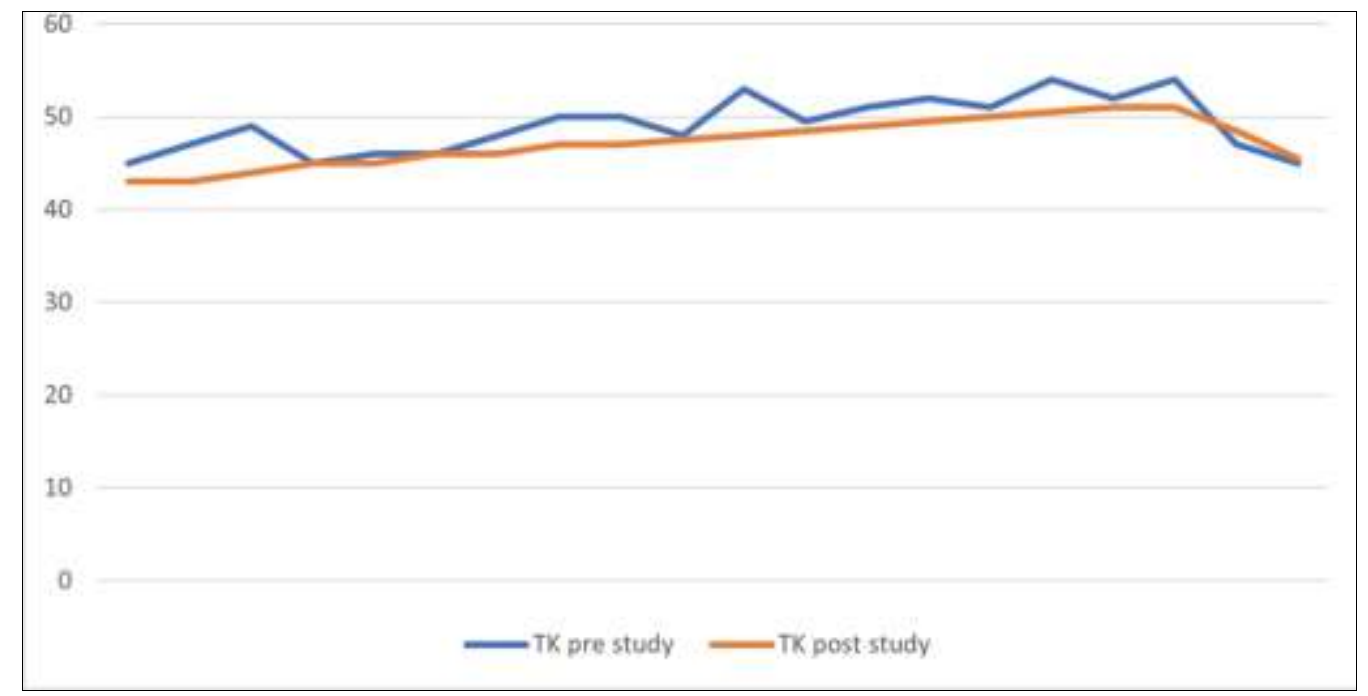

Graph 1: Thoracic kyphosis angles in the study group

Table 1: Demographic characteristics of patient

\begin{tabular}{|c|c|c|c|}
\hline & Study group $(\mathbf{n}=\mathbf{4 0})$ & Control group $(\mathbf{n}=\mathbf{4 0})$ & P value \\
\hline Age (years) & $68.16+/-19.45$ & $66.22+/-24.28$ & 0.9167 \\
\hline Sex, $\mathrm{n}(\mathrm{male} / \mathrm{female})$ & $33 / 47$ & $35 / 45$ & 0.576 \\
\hline BMI $\left(\mathrm{kg} / \mathrm{m}^{2}\right)$ & $21.81+/ 4.45$ & $22.14+/-4.13$ & 0.33 \\
\hline Symptoms (months) & $89+/-43$ & $85+/-57$ & 0.373 \\
\hline Working status $(\mathrm{n},(\%))$ & & & 0.418 \\
\hline Sedentary & 33 & 31 & \\
\hline Mild/mod & 5 & 8 & \\
\hline Heavy working & 2 & 1 & \\
\hline
\end{tabular}

Table 2: Clinical parameters of both the groups

\begin{tabular}{|c|c|c|c|c|}
\hline Parameter & Point of reference & Study group & Control group & P value \\
\hline & Initial & $49.12+/-4.53$ & $48.75+/-3.75$ & 0.33 \\
\hline & After 3 months & $47.3+/-5.12$ & $48.05+/-4.23$ & \\
\hline & & 0.019 & 0.16 & \\
\hline & Initial & $34.05+/-4.3$ & $32.75+/-3.7$ & 0.184 \\
\hline & After 3 months & $32.2+/-3.7$ & $31.15+/-3.4$ & \\
\hline & & 0.056 & 0.116 & \\
\hline Thoracic kyphosis & Initial & $45.88+/-5.85$ & $44.75+/-3.75$ & 0.097 \\
\hline & After 3 months & $44.58+/-4.98$ & $44.05+/-$ & \\
\hline & & 0.078 & 0.164 & \\
\hline
\end{tabular}

Table 3: Radiological parameters of both the groups

\begin{tabular}{|c|c|c|c|c|}
\hline Parameter & Point of reference & Study group & Control group & P value \\
\hline VAS (Pain) & Initial & $4.97+/-3.14$ & $5.17+/-3.01$ & 0.36 \\
\hline & After 3 months & $3.97+/-2.89$ & $4.92+/-2.98$ & -.052 \\
\hline & & 0.016 & 0.25 & \\
\hline ODI & Initial & $26.59+/-11.56$ & $24.82+/-14.03$ & 0.184 \\
\hline & After 3 months & $20.15+/-9.87$ & $23.82+/-13.89$ & 0.031 \\
\hline & & 0.003 & 0.313 & \\
\hline MST & Initial & $14.24+/-1.19$ & $14.21+/-1.45$ & 0.11 \\
\hline & After 3 months & $14.83+/-2.11$ & $14.28+/-1.06$ & 0.04 \\
\hline Occiput wall & & 0.039 & 0.78 & \\
\hline Distance $(\mathrm{cm})$ & Initial & $3.62+/-1.7$ & $3.19+/-1.17$ & 0.15 \\
\hline & After 3 months & $2.86+/-1.2$ & $2.98+/-1.45$ & 0.367 \\
\hline
\end{tabular}

\section{Discussion}

Posture is defined as the relative order of the body parts ${ }^{[15]}$. Cervical lordosis, thoracic kyphosis, and lumbar lordosis are the major components of the physiological curvatures of the spine in the sagittal plane. Whenever these curvatures are disturbed, this leads to postural deviation. Bad posture leads to exaggerated joint load and inadequate tension in the soft tissues, leading to pain and functional loss ${ }^{[16]}$.

It has long been speculated that improvement in back extensor strength can lead to postural improvement or other parameters of hyperkyphosis, such as spinal extension mobility, improved postural awareness, or fewer incident vertebral 
column fractures ${ }^{[1]}$.

A recent systematic review by Bansal et al. has supported the need of a well-designed RCT which can examine the effects of exercise programme on patients of Hyperkyphosis ${ }^{[1]}$. As many of these cases have a background of osteoporosis behind them, it is even more important to identify a more comprehensive assessment of health and functional outcome in elderly kyphotic population. This should also include impact and treatment effects. This further validates the need to study effects of exercises which is an easy and safe option for such individuals ${ }^{[11]}$.

The purpose of this study was to find the association between targeted spinal exercises and clinic-radiological improvement in patients of Hyperkyphosis in our geographical area. The fact that we could not find any study pertaining to our area highlighted the need of this study.

Our review of the literature has been suggestive that evidence of effects of exercise on age-related hyper kyphosis is scarce and largely un applicable to our part of the world, although few trials report some benefit of exercise or targeted interventions that include exercise in patients of hyperkyphosis ${ }^{[12-13]}$.

Our study was conducted across both the groups that had a comparable distribution of age, gender, BMI, working status of the patient at the time of enrolment and duration of symptoms. This was important as variation in any of these variables could have caused a confounding implication on the rest of the values.

Results of clinical and radiological parameters in our study showed significantly better improvement in Thoracic kyphosis angles on X-rays, Occiput to wall distance, Modified Schoeber test scores and subjective scores such as ODI and VAS Scores. Results of our parameters of Thoracic kyphosis in best erect posture and Lumbar lordosis were not statistically significant but correlated with other results.

Panjabi et al. ${ }^{[18]}$ reported the role of lumbar stabilization exercise in providing dynamic stabilization in the segments providing lumbar segmental stability. They also found it to be useful strategy in decreasing spinal functional disorder. Richardson et al. ${ }^{[19]}$ proposed that exercises directing in lumbar stabilization also increased the spinal stability and pelvic stability in performing functional postures and movements. These exercises have also been found to be effective in reducing low back pain, increasing the range of motion of the trunk and pelvis, and improving body balance [20, 21].

It has been observed that the Cobb angle progresses slowly with age to the tune of less than $1^{\circ}$ a year ${ }^{[22]}$. This indicates that if treatment is effectively maintained over a period, reducing kyphosis by even a small amount may be especially important. The change in kyphosis is consistent with previous studies reporting improvement in clinical measures of kyphosis after targeted spine strengthening ${ }^{[7,23]}$. The 1.8 degrees reduction in thoracic kyphosis because of intervention seen in our study also indicates the reduction in magnitude which might be expected of an associated vertebral fracture.

Kamali et al. [26] evaluated 18 -30-year-old patients with kyphosis over 45 degrees of kyphosis by methods of manual therapy, mobilization, massage, and myofascial release in one group and stretching and strengthening of back muscle in another group. They found angle of thoracic kyphosis was smaller and back extensor muscle strength was significantly greater in both the exercise and manual therapy groups $(\mathrm{p}<$ 0.001).

Few other studies ${ }^{[27-30]}$ have also evaluated the role of physical therapy and exercises in patients of hyperkyphosis and found that there was favourable result in kyphosis and lordosis seen in patients of Hyperkyphosis when targeted exercise programmes were initiated. Many of these studies have however measured kyphosis by other tools such as inclinometer, Kyphometer, Flexicurve or photometric technique.

We decided to measure thoracic kyphosis by Cobb's angle on Xray which has so far been the most accurate estimation. The difference after intervention in study group of 1.8degrees exceeded the 1.4-degree standard error of the measurement and was statistically significant but the difference in change in Cobb angle did not reach the minimal detectable difference of 2.6 degrees. As lumbar lordosis also changes following the change in kyphosis, we also tried to evaluate change in lordosis in our study group. Few studies earlier ${ }^{[30-32]}$ have found a favourable relation between lumbar lordosis but these studies have been conducted over a long period of time and used other methods such as inclinometer. We in our study did not find lumbar lordosis to show significantly different values but the values did improve. The mean lordotic angles could also have been affected with other parameters and we intend to study this in greater detail in future research.

While we did find change in the clinical measure of kyphosis, a longer period may also be needed before radiographic changes are established. It is also possible that differences in the superior landmarks used to assess kyphosis, such as the T2-3 interspace in the kyphometer-derived kyphosis, and the upper endplate of $\mathrm{T} 4$ in the radiographic Cobb angle of kyphosis, may confound these measurements as T23 interspace may be more anterior than $\mathrm{T} 4$ in the sagittal plane [33].

We also tried to evaluate the kyphosis in the best erect posture, a parameter stressed by Bansal et al. ${ }^{[1]}$ to be important while assessing kyphotic improvement. Though our found a significant improvement in kyphosis angle with exercise programme in relaxed posture, it was not the case in best erect posture. In the best erect posture, the kyphosis as evaluated pre- and post-study revealed no significant difference, indicating that exercise regimen probably only helps to correct the already correctable kyphosis component in these patients. As there are no earlier studies detailing this issue, we recommend more studies on this parameter in future.

There are no prior studies that specifically measure clinical kyphosis as measured by occiput to wall (OTW) distance or the effect of exercise on this parameter. We found this to be a quantifiable and easy tool to measure which mirrored the other results. However, the $1.9-\mathrm{cm}$ change in OTW distance observed in our study did not exceed the smallest detectable difference of $3.2 \mathrm{~cm}$ according to Bland-Altman criteria previously reported among adults with an kylosing spondylitis $[24,25]$

Though lumbar flexibility can be evaluated by range of motion, it is a poor parameter due to high variability, interobserver variation and confounding by other temporary factors. Modified Schoeber test has been a function of lumbar flexibility in literature ${ }^{[34]}$ and through this parameter our study also showed significant improvement in Lumbar range of motion as significantly higher $(\mathrm{p}=0.039)$ Schober test scores were reported with exercises. This has also been previously validated by Filiz et al. ${ }^{[35]}$

Improvement in VAS and ODI scores showed a better subjective and functional improvement of patients. This has been correlated in previous studies also to better self-esteem 
and function. Earlier studies ${ }^{[1,35,36]}$ have also found these parameters to be positively impacted by targeted muscle strengthening.

Our study has several limitations. The multiple causation of Hyperkyphosis could create a bias which could have gone unnoticed. A placebo if applied to the control group, could have produced better results in that group. To be able to draw firm conclusions about the efficacy of such physical therapy, a placebo-controlled trial should be designed. The short follow-up period limits our results to the short term only. Also, the medications that might have been prescribed to the patients, their period of use and at which doses, and the adherence of the patients to the exercise schedule were not recorded. Therefore, the possible contribution of the medications and exercises on the outcome parameters could not be estimated.

In conclusion, despite certain limitations and short treatment duration, the results of the present study revealed that exercises regimen favourably impacted clinical and radiological parameters of kyphosis in hyperkyphotic individuals. It was accompanied with better self-image, function, and reduced pain. As even a minor change in these individuals may cause far reaching effects, we feel that a further randomized controlled long-term studies are needed to confirm these results.

\section{Conclusion}

The results of our study match with the results found in the studies done elsewhere comparing the results of thoracic kyphosis in hyperkyphotic individuals when subjected to physical therapy including targeted exercise programmes. Though further studies are warranted to examine the finer aspects and look at a larger data over a longer period, we suggest progressive specific targeted exercise regimes focussing on spinal extension and muscle strengthening of back to be advised in every patient presenting with hyperkyphosis keeping in mind especially the other morbidities seen usually in such patients

\section{References}

1. Bansal S, Katzman WB, Giangregorio LM. Exercise for improving age-related hyperkyphotic posture: a systematic review. Arch Phys Med Rehabil 2014;95(1):129-140. doi:10.1016/j.apmr.2013.06.022

2. Kado DM, Huang MH, Karlamangla AS, Barrett-Connor E, Greendale GA. Hyperkyphotic posture predicts mortality in older community-dwelling men and women: a prospective study. J Am Geriatr Soc 2004;52:1662-7.

3. Takahashi T, Ishida K, Hirose D et al. Trunk deformity is associated with a reduction in outdoor activities of daily living and life satisfaction in community-dwelling older people. Osteoporosis Int 2005;16:273-9.

4. Teramoto S, Suzuki M, Matsuse T et al. [Influence of kyphosis on the age-related decline in pulmonary function] [Japanese] Nihon Ronen Igakkai Zasshi 1998;35:23-7.

5. Ryan SD, Fried LP. The impact of kyphosis on daily functioning. J Am Geriatr Soc 1997;45:1479-86.

6. Kado DM, Huang MH, Nguyen CB, Barrett-Connor E, Greendale GA. Hyperkyphotic posture and risk of injurious falls in older persons: the Rancho Bernardo Study. J Gerontol A Biol Sci Med Sci 2007;62:652-7.

7. Katzman WB, Sellmeyer DE, Stewart AL, Wanek L, Hamel KA. Changes in flexed posture, musculoskeletal impairments, and physical performance after group exercise in community-dwelling older women. Arch Phys Med Rehabil 2007;88:192-9.

8. Katzman WB, Wanek L, Shepherd JA, Sellmeyer DE. Age-related hyperkyphosis: its causes, consequences, and management. J Orthop Sports Phys Ther 2010;40:352-60.

9. Balzini L, Vannucchi L, Benvenuti F et al. Clinical characteristics of flexed posture in elderly women. J Am Geriatr Soc 2003;51:1419-1426.

10. Lorbergs AL, Murabito JM, Jarraya M, et al. Thoracic Kyphosis and Physical Function: The Framingham Study. J Am Geriatr Soc 2017;65(10):2257-2264. doi:10.1111/jgs. 15038

11. Bayliss M, Miltenburger C, White M, Alvares L. A conceptual and disease model framework for osteoporotic kyphosis. Osteoporos Int 2013;24(9):2423-2432. doi: 10.1007/s00198-013-2317-6.

12. Kado D, Browner W, Palmero L, Nevitt M, Genant H, Cummings S. Vertebral fractures and mortality in older women. A prospective study. Arch Intern Med 1999;159:1215-1220.

13. Katzman WB, Sellmeyer DE, Stewart AL, Wanek L, Hamel KA. Changes in flexed posture, musculoskeletal impairments, and physical performance after group exercise in community-dwelling older women. Arch Phys Med Rehabil 2007;88(2):192-199. doi: 10.1016/j.apmr.2006.10.033.

14. Katzman WB, Gladin A, Lane NE et al. Feasibility and Acceptability of Technology-Based Exercise and Posture Training in Older Adults With Age-Related Hyperkyphosis: Pre-Post Study. JMIR Aging 2019;2(1):e12199. doi:10.2196/12199

15. Kaijser Alin C, Uzunel E, Grahn Kronhed AC, Alinaghizadeh $\mathrm{H}$, Salminen $\mathrm{H}$. Effect of treatment on back pain and back extensor strength with a spinal orthosis in older women with osteoporosis: a randomized controlled trial. Arch Osteoporos 2019;14(1):5. Published 2019 Jan 9. doi:10.1007/s11657-018-0555-0

16. Czaprowski D, Stolinski L, Tyrakowski M, Kozinoga M, Kotwicki T. Non-structural misalignments of body posture in the sagittal plane. Scoliosis Spinal Disord 2018;13:6. doi: 10.1186/s13013-018-0151-5.

17. Christie HJ, Kumar S, Warren SA. Postural aberrations in low back pain. Arch Phys Med Rehabil 1995;76:218-24. doi: 10.1016/S0003-9993(95)80604-0.

18. Panjabi MM: Clinical spinal instability and low back pain. J Electromyogr Kinesiol 2003;13:371-379.

19. Richardson CA, Snijders CJ, Hides JA et al. The relation between the transversus abdominis muscles, sacroiliac joint mechanics, and low back pain. Spine 2002;27:399405.

20. Kirkesola G. Sling exercise therapy (S-E-T): a total concept for exercise and active treatment of musculoskeletal disorders. J Korean Orthop Man Phys Ther, 2001;7:87-106.

21. Carpes FP, Reinehr FB, Mota CB: Effects of a program for trunk strength and stability on pain, low back and pelvis kinematics, and body balance: a pilot study. $\mathbf{J}$ Bodyw Mov Ther 2008;12:22-30.

22. Kado DM, Huang MH, Karlamangla AS, Cawthon P, Katzman W, Hillier TA et al. Factors associated with kyphosis progression in older women: 15 years' experience in the study of osteoporotic fractures. J Bone Miner Res 2013;28(1):179-187. doi: 10.1002/jbmr.1728.

23. Greendale GA, Huang MH, Karlamangla AS, Seeger L, Crawford S. Yoga decreases kyphosis in senior women 
and men with adult-onset hyperkyphosis: results of a randomized controlled trial. J Am Geriatr Soc 2009. doi: 10.1111/j.1532-5415.2009.02391.

24. Calvo-Gutiérrez J, Garrido-Castro J, González-Navas C, Castro-Villegas M, Ortega-Castro R, López-Medina C. Inter-rater reliability of clinical mobility measures in ankylosing spondylitis. BMC Musculoskelet Disord 2016;17(1):382. doi: 10.1186/s12891-016-1242-1.

25. Katzman WB, Gladin A, Lane NE, et al. Feasibility and Acceptability of Technology-Based Exercise and Posture Training in Older Adults With Age-Related Hyperkyphosis: Pre-Post Study. JMIR Aging. 2019;2(1):e12199. doi:10.2196/12199

26. Kamali F, Shirazi SA, Ebrahimi S, Mirshamsi M, Ghanbari A. Comparison of manual therapy and exercise therapy for postural hyperkyphosis: A randomized clinical trial. Physiother Theory Pract 2016;32:92-7. 10.3109/09593985.2015.1110739

27. Muyor JM, López-Miñarro PA, Casimiro AJ. Effect of stretching program in an industrial workplace on hamstring flexibility and sagittal spinal posture of adult women workers: a randomized controlled trial. J Back Musculoskelet Rehabil 2012;25(3):161-9. doi: 10.3233/BMR-2012-0323. PMID: 22935854.

28. Seidi F, Rajabi R, Ebrahimi I, Alizadeh MH, Minoonejad $\mathrm{H}$. The efficiency of corrective exercise interventions on thoracic hyper-kyphosis angle. J Back Musculoskelet Rehabil 2014;27(1):7-16. doi: 10.3233/BMR-130411. PMID: 23948845.

29. Jang HJ, Hughes LC, Oh D-W, Kim SY. Effects of Corrective Exercise for Thoracic Hyperkyphosis on Posture, Balance, and Well-Being in Older Women: A Double-Blind, Group-Matched Design. J Geriatr Phys Ther 2017; 13:1-11.

30. Fatemi R, Javid M, Najafabadi EM. Effects of William training on lumbosacral muscles function, lumbar curve and pain. J Back Musculoskelet Rehabil 2015;28:591-7. 10.3233/BMR-150585

31. Hosseinifar M, Ghiasi F, Akbari A, Ghorbani M. The effect of stabilization exercises on lumbar lordosis in patients with low back pain. Ann Trop Med Public Heal 2017;10:1779-84.

32. Kwang-Jun K, Gi-Chul H, Yook YS, Kang SJ. Effects of 12-week lumbar stabilization exercise and sling exercise on lumbosacral region angle, lumbar muscle strength, and pain scale of patients with chronic low back pain. J Phys Ther Sci. 2018;30:1-22. 10.1589/jpts.30

33. Katzman WB, Parimi N, Gladin A, et al. Sex differences in response to targeted kyphosis specific exercise and posture training in community-dwelling older adults: a randomized controlled trial. BMC Musculoskelet Disord 2017;18(1):509. Published 2017. doi:10.1186/s12891017-1862-0

34. Robinson HS, Mengshoel AM. Assessments of lumbar flexion range of motion: intertester reliability and concurrent validity of 2 commonly used clinical tests.Spine (Phila Pa 1976) 2014;39(4):E270-5.

35. Filiz MB, Firat SC. Effects of Physical Therapy on Pain, Functional Status, Sagittal Spinal Alignment, and Spinal Mobility in Chronic Non-specific Low Back Pain. Eurasian J Med 2019;51(1):22-26. doi:10.5152/eurasianjmed.2018.18126

36. González-Gálvez N, Gea-García GM, Marcos-Pardo PJ. Effects of exercise programs on kyphosis and lordosis angle: A systematic review and meta-analysis. PLoS One 2019;14(4):e0216180. Published

2019. 